J. Lake Sci. (湖泊科学) , 2019, 31(5): 1229-1238

DOI 10. 18307/2019. 0506

(c) 2019 by Journal of Lake Sciences

\title{
改性黑臭河道沉积物对营养盐去除的研究
}

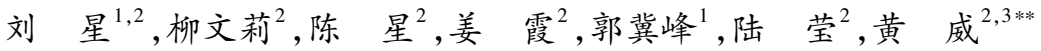 \\ (1: 长安大学建筑工程学院, 旱区地下水文与生态效应教育部重点实验室, 西安 710054) \\ (2: 中国环境科学研究院, 湖泊水污染治理与生态修复技术国家工程实验室, 北京 100012) \\ (3: 东华大学环境科学与工程学院,上海 201620)
}

\begin{abstract}
摘 要: 为研究黑臭河道沉积物资源化利用方式, 评估沉积物资源化以后对营养盐去除效果和研究其去除机制, 以北京 市某黑臭河道沉积物 (S) 为供试样品, 对其进行氧化并负载金属离子改性, 最终得到氧化一载钠改性沉积物 (MS). 选取 S 和 MS 两种材料, 评估其对氮和磷的去除效果, 通过采用动力学、热力学等手段, 进一步阐明改性材料对氮、磷去除机制. 结果表明, 在环境温度为 $35^{\circ} \mathrm{C}$, 固液比 $1: 50(\mathrm{~g}: \mathrm{ml})$ 的条件下, 经氧化一载钠联合改性材料对氮 (初始浓度 $20 \mathrm{mg} / \mathrm{L}$ ) 的 去除效果均达到 $90 \%$ 以上, 其中对磷 (初始浓度 $10 \mathrm{mg} / \mathrm{L}$ ) 的去除率最高可达 $98 \%$; 准二级动力学模型能更好的描述改性 材料的吸附动力学行为 $\left(R^{2}>0.93\right)$, 且动力学和吸附等温分析结果显示, Langmuir 模型可以更好地描述两种材料的吸附 等温线, 改性材料对氮和磷的最大吸附量分别达到 0.891 和 $0.474 \mathrm{mg} / \mathrm{g}$; 热力学研究结果表明, 吸附过程中, 标准反应焓 变 $\left(\Delta H^{0}\right)$ 、标准反应熵变 $\left(\Delta S^{0}\right)$ 均为正值,标准吉布斯自由能变 $\left(\Delta G^{0}\right)$ 为负值, 且随着温度的升高, $\Delta G^{0}$ 呈下降趋势. 研究 显示, MS 具有更高的吸附性能, 对氮、磷的吸附主要是材料表面活性吸附点位起主导作用, 并且吸附过程是个吸热、自发 进行的过程, 更高的温度更有利于吸附. 在同一温度下, 随着氮、磷初始浓度的增加, MS 对氮、磷的解吸比吸附过程更容 易发生.
\end{abstract}

关键词: 黑臭河道;改性;沉积物;营养盐;吸附

\section{Efficiency and mechanism of nutrient removal using modified sediments from a malodor- ous river}

\author{
LIU Xing ${ }^{1,2}$, LIU Wenli ${ }^{2}$, CHEN Xing ${ }^{2}$, JIANG Xia ${ }^{2}$, GUO Jifeng ${ }^{1}$, LU Ying ${ }^{2} \&$ HUANG Wei ${ }^{2,3 * *}$ \\ (1: School of Architecture and Engineering, Key Laboratory of Subsurface Hydrology and Ecology in Arid Areas, Ministry of \\ Education, Chang'an University, Xi'an 710054, P.R.China) \\ (2: National Engineering Laboratory for Lake Pollution Control and Ecological Restoration, Chinese Research Academy of En- \\ vironmental Sciences, Beijing 100012, P.R.China) \\ (3: College of Environmental Science and Engineering, Donghua University, Shanghai 201620, P.R. China)
}

\begin{abstract}
In order to study the method of sediment resource utilization in the malodorous river, and to evaluate the nutrient removal efficiency using modified sediment and its removal mechanism, the sediment samples $(S)$ from a seriously malodorous river in Beijing were collected in this study. The sediment was modified by oxidation and metal ion-loaded (MS). The nutrient removal efficiency for S and MS was evaluated. The removal mechanism of nutrient was studied through kinetics, equilibrium, and thermodynamic experiments. The results indicated that when the environmental temperature was $35^{\circ} \mathrm{C}$, and solid to liquid ratio was $1: 50$ $(\mathrm{g}: \mathrm{ml})$, the nitrogen (initial concentration, $20 \mathrm{mg} / \mathrm{L}$ ) and phosphorus (initial concentration, $10 \mathrm{mg} / \mathrm{L}$ ) removal efficiency by using MS was up to $90 \%$ and $98 \%$, respectively. The pseudo-second-order model could better describe the sorption kinetics $\left(R^{2}>\right.$ 0.93 ), and the kinetic and isotherm study illustrated that data from the isotherm experiments were well described by Langmuir isotherm model. The sorption capacity reached $0.891 \mathrm{mg} / \mathrm{g}$ for nitrogen and $0.474 \mathrm{mg} / \mathrm{g}$ for phosphorus, respectively. The thermodynamic result suggested that enthalpy $\left(\Delta H^{0}\right)$ and entropy $\left(\Delta S^{0}\right)$ were positive, and Gibbs energy $\left(\Delta G^{0}\right)$ was negative during the
\end{abstract}

* 北京市自然科学基金项目 (8174080) 和中国博士后科学基金项目 (2017M610968) 联合资助. 2018-12-16 收稿; 2019-02-27 收修改稿. 刘星(1995 ), 男,硕士研究生;E-mail: m17602924013@163.com.

** 通信作者;E-mail : yixinghd6@163.com. 
adsorption experiments. The values of $\Delta G^{0}$ decreased with the increase of temperature. The results showed that MS had the higher nutrient sorption capacity, and the exterior activated site had the main function of phosphorus and nitrogen sorption. The sorption process was a spontaneous or endothermic reaction, and high temperature was in favour of sorption. In addition, the results also indicated that desorption occurred more easily than sorption at the same temperature.

Keywords: Malodorous river; modified; sediments; nutrient; adsorption

河道黑臭是我国城市环境污染和生态破坏的代表性恶果. 目前许多城市根据自身的特点, 开展了许多 黑臭河道整治工程, 基本遵循“控源一净化一修复” 的思路 ${ }^{[1]}$. 然而, 由于城市黑臭河道是一窄浅型水体, 其边 界条件极其复杂, 沉积物沉积年代久远, 随着相应黑臭河道治理工程的实施, 污染负荷得到了大幅去除、黑 臭逐步好转,黑臭河道沉积物的处置问题凸显出来 ${ }^{[2]}$. 现阶段主要通过异位处理技术, 大规模疏浚黑臭河道 沉积物后, 采用淋洗, 焚烧、高温高压氧化、玻璃化、热解等技术 ${ }^{[3]}$, 降低沉积物中污染物含量, 使处理后的沉 积物能进一步得到应用. 通常处理后的沉积物应用在路基、建筑材料等领域. 但是, 由于处理后的沉积物在 其资源化利用领域并不占有成本和性能的优势, 因此, 黑臭河道沉积物处理处置体系得不到最终完善及大 规模应用. 探寻合适途径对黑臭河道沉积物进行再利用有着重大的意义.

表面修饰是通过吸附、涂敷、包覆、材料微粒表面与改性剂之间的反应等作用,对材料微粒进行表面改 性, 改变材料表面结构或物质组成等, 以提高材料的吸附性能, 其中通过表面修饰手段提高材料对营养盐去 除率的研究已被大量报导 ${ }^{[4-5]}$. 疏浚后的黑臭河道沉积物经高温处理后具有较高的比表面积、孔隙率等方面 优势, 具备作为吸附材料的潜质 ${ }^{[6]}$. 氮和磷作为我国绝大部分河湖营养的主要限制因子, 经过不同途径输人 到河道等水体中, 过量的氮和磷使水体富营养化, 导致水体的透明度下降, 影响水体美观; 其次使得水体中 溶解氧浓度下降, 水生生态系统中的很多其他物种死亡, 破坏了生态系统的平衡 ${ }^{[-9]}$. 在早期的研究中, 一些 高效的脱氮除磷方法已经被报道. 土壤、炉渣、沸石和方解石成为了去除水体中磷的常用材料,沸石可作为 用于脱氮的高效吸附剂 ${ }^{[10-11]}$; 部分研究也表明,一些改性材料, 如负载氧化铁、铝或者锰的烧黏土也是效果 极佳的磷吸附剂 ${ }^{[12-13]}$. 但是在这些小型的实例系统中, 能同时达到经济、高效、环保等优点较为困难. 因此, 以黑臭河道沉积物为原料,经过表面修饰等手段,开展新型材料对氮、磷去除的研究尤为重要.

本研究拟选取北京市某黑臭河道黑臭现象较为严重段 $(1.65 \mathrm{~km})$ 为研究对象, 通过对沉积物进行氧化 和负载金属离子的手段制备新型材料, 评估所制备的材料对水中氨氮和磷酸盐的去除效果, 阐明材料对氨 氮和磷酸盐的去除机制,为黑臭河道沉积物资源化途径和利用领域提供一定的理论依据.

\section{1 材料与方法}

\section{1 样品采集与改性材料的制备}

本研究采集黑臭水体污染严重段表层 $20 \mathrm{~cm}$ 沉积物样,在 $1.65 \mathrm{~km}$ 河段内每 $300 \mathrm{~m}$ 采集表层沉积物(采 集次数 $n=5)$, 混匀后 $24 \mathrm{~h}$ 内带回实验室, 取适量沉积物样品于冷冻干燥机中冻干 (4d), 然后将冻干后的沉 积物样品剔除砂石等杂质,研磨过篮 (100 目), 装人自封袋中用于物化性质测定和材料改性实验. 沉积物样 品经冷冻干燥后, 均用 $\mathrm{pH}=1.0$ 的酸进行浸泡润洗 (去除表面灰分和有机质)、过滤、烘干, 所得原材料标记 为 $\mathrm{S}$. 采用氧化一载钠联用手段对沉积物原材料 $(\mathrm{S})$ 进行改性, 具体步骤如下: 采用 $2.5 \mathrm{~mol} / \mathrm{L} \mathrm{HNO}_{3}$, 以 $1: 20$ 固液比, 进行氧化改性, 在室温下经 $12 \mathrm{~h}$ 充分混匀振荡, 然后过滤, 放置于 $60^{\circ} \mathrm{C}$ 烘箱中 $12 \mathrm{~h}$ 烘干, 得到氧化 改性后的沉积物; 取氧化改性沉积物, 采用 $0.20 \mathrm{~mol} / \mathrm{L} \mathrm{NaCl}$ 溶液, $\mathrm{pH}$ 为 $2.5 \sim 3.5$, 以 $1: 20$ 固夜比, 进行载钠 改性, 依旧在室温下经 $12 \mathrm{~h}$ 充分混匀振荡, 过滤, 放置于 $60^{\circ} \mathrm{C}$ 烘箱中 $12 \mathrm{~h}$ 烘干, 得到载钠改性后沉积物, 标 记为 $\mathrm{MS}^{[14]}$. 通过氧化一负载 $\mathrm{Na}^{+}$, 使材料表面形成氧化膜,一方面可增加沉积物比表面积,另一方可提供更 多的活性吸附点位, 从而提高氮、磷去除率.

\section{2 常规指标测定}

沉积物中总磷 (TP) 、总氮 (TN) 采用欧洲标准测试方法 (SMT) 和分级浸提法进行测 ${ }^{\text {定 }}{ }^{[15-16]}$; 通过质量与 体积比 $1: 2.5$ 的泥和水混合后测定浑浊液中的 $\mathrm{pH}^{[17]}$; 有机质 $(\mathrm{OM})$ 含量通过样品在 $550^{\circ} \mathrm{C}$ 下煅烧 $4 \mathrm{~h}$, 测定 样品煅烧前后质量差得出 ${ }^{[18]}$. 采用 Micrometritics Tristar 3000 表面分析仪, 在 $0.001 \sim 0.995$ 大气压下通过氮 
气吸脱附技术测定材料比表面积. 样品的氧化物成分通过 X 射线荧光分析仪 (S4 EXPLORER, 德国) 来测 定. 河道沉积物和材料样品的主要物化性质如表 1 和表 2 所示.

\section{3 实验设计}

1.3.1 效果评估实验 称取 $\mathrm{S}$ 和 MS 样品 $1 \mathrm{~g}$, 分别加人盛有 $50 \mathrm{ml}$ 初始浓度为 $10 \mathrm{mg} / \mathrm{L}$ 磷溶液( 用 $105^{\circ} \mathrm{C}$ 下烘 干 $2 \mathrm{~h}$ 的 $\mathrm{KH}_{2} \mathrm{PO}_{4}$ 配制) 和 $20 \mathrm{mg} / \mathrm{L}$ 氨氮溶液的三角瓶中 (用 $105^{\circ} \mathrm{C}$ 下烘干 $2 \mathrm{~h}$ 的 $\mathrm{NH}_{4} \mathrm{Cl}$ 配制). 然后将所有 三角瓶用保鲜膜密封放置于摇床以 220 转/ $\min$ 振荡, 在 $480 \mathrm{~min}$ 时进行取样, 过滤 $(0.45 \mu \mathrm{m})$, 测定样品中 磷浓度和氨氮浓度. 本研究设置 3 个温度梯度 $\left(15 、 25\right.$ 和 $\left.35^{\circ} \mathrm{C}\right)$ 来进行改性沉积物对氨氮和磷酸盐去除效果 评估的实验, 实验设置 3 组平行.

1.3.2 吸附动力学实验 称取 $\mathrm{S}$ 和 MS 样品 $1 \mathrm{~g}$, 分别加人盛有 $50 \mathrm{ml}$ 初始浓度为 $10 \mathrm{mg} / \mathrm{L}$ 磷溶液( 用 $105^{\circ} \mathrm{C}$ 下烘 干 $2 \mathrm{~h}$ 的 $\mathrm{KH}_{2} \mathrm{PO}_{4}$ 配制) 和 $20 \mathrm{mg} / \mathrm{L}$ 氨氮溶液的三角瓶中 (用 $105^{\circ} \mathrm{C}$ 下烘干 $2 \mathrm{~h}$ 的 $\mathrm{NH}_{4} \mathrm{Cl}$ 配制). 然后将所有三 角瓶用保鲜膜密封放置于摇床中分别在 $15 、 25$ 和 $35^{\circ} \mathrm{C}$ 状态下以 220 转 $/ \min$ 振荡, 分别在 $0 、 1 、 2 、 4 、 8 、 10 、 20 、$ $40 、 60 、 120 、 240 、 360$ 和 $480 \mathrm{~min}$ 时进行取样, 过滤 $(0.45 \mu \mathrm{m})$, 测定样品中磷和氨氮浓度, 实验设置 3 组平行.

表 1 河水水质参数、河道沉积物的物化性质 (均值 \pm 标准误差)

Tab.1 Physical and chemical properties of river and sediment ( mean \pm standard error)

\begin{tabular}{cccccc}
\hline \multirow{2}{*}{ 沉积物 } & $\mathrm{TP} /(\mathrm{mg} / \mathrm{kg})$ & $\mathrm{TN} /(\mathrm{mg} / \mathrm{kg})$ & $\mathrm{OM} / \%$ & 含水率 $/ \%$ & $\mathrm{pH}$ \\
\cline { 2 - 6 } & $1845.3 \pm 167.5$ & $3892.1 \pm 273.12$ & $10.67 \pm 1.23$ & $75.22 \pm 12.32$ & $8.37 \pm 0.71$ \\
\hline \multirow{2}{*}{ 河水 } & $\mathrm{TP} /(\mathrm{mg} / \mathrm{L})$ & $\mathrm{TN} /(\mathrm{mg} / \mathrm{L})$ & $\mathrm{NH}_{3}-\mathrm{N} /(\mathrm{mg} / \mathrm{L})$ & $\mathrm{DO} /(\mathrm{mg} / \mathrm{L})$ & $\mathrm{pH}$ \\
\cline { 2 - 6 } & $0.89 \pm 0.08$ & $9.31 \pm 1.05$ & $6.48 \pm 0.55$ & $0.40 \pm 0.12$ & $8.18 \pm 0.66$ \\
\hline
\end{tabular}

表 2 沉积物材料改性前后的物化性质

Tab.2 Physical and chemical properties of sediment materials before and after modification

\begin{tabular}{|c|c|c|c|c|c|c|c|c|c|}
\hline \multirow{2}{*}{ 材料 } & \multicolumn{2}{|c|}{ 表面性质 } & \multicolumn{5}{|c|}{ 氧化物含量/ $\%$} & \multicolumn{2}{|c|}{ 物理性质 } \\
\hline & 比表面积 $/\left(\mathrm{m}^{2} / \mathrm{g}\right)$ & 孔径 $/\left(\mathrm{cm}^{3} / \mathrm{g}\right)$ & $\mathrm{SiO}_{2}$ & $\mathrm{Na}_{2} \mathrm{O}$ & $\mathrm{Al}_{2} \mathrm{O}_{3}$ & $\mathrm{MgO}$ & $\mathrm{CaO}$ & $\mathrm{OM} / \%$ & $\mathrm{pH}$ \\
\hline $\mathrm{S}$ & 2.83 & $8.89 \times 10^{-3}$ & 62.5 & 0.43 & 17.9 & 1.56 & 0.87 & 7.84 & 7.35 \\
\hline MS & $30.07 \pm 1.82$ & $6.89 \times 10^{-2}$ & 59.8 & 2.29 & 21.40 & 1.52 & 0.17 & 5.89 & 7.39 \\
\hline
\end{tabular}

1.3.3 吸附等温及吸附热力学实验 称取 $\mathrm{S}$ 和 MS 样品 $1 \mathrm{~g}$, 分别加人盛有 $50 \mathrm{ml}$ 不同初始浓度 $(0 、 1 、 2 、 5 、 10$ 、 20 和 $50 \mathrm{mg} / \mathrm{L}$ ) 磷溶液 (用 $105^{\circ} \mathrm{C}$ 下烘干 $2 \mathrm{~h}$ 的 $\mathrm{KH}_{2} \mathrm{PO}_{4}$ 配制) 和氨氮溶液的三角瓶中 (用 $105^{\circ} \mathrm{C}$ 下烘干 $2 \mathrm{~h}$ 的 $\mathrm{NH}_{4} \mathrm{Cl}$ 配制). 然后将所有三角瓶用保鲜膜密封放置于摇床中分别在 $15 、 25$ 和 $35^{\circ} \mathrm{C}$ 状态下以 220 转/ $\mathrm{min}$ 振 荡 $480 \mathrm{~min}$ 达到吸附平衡. 通过拟合吸附等温公式确定吸附等温各项参数; 采用线性方程拟合热力学公式, 确定在不同浓度下获得的热力学参数如焓 $(\Delta H)$, 吉布斯能源 $(\Delta G)$ 和熵值 $(\Delta S)$. 实验设置 3 组平行.

\section{4 数据处理}

材料不同时间点磷和氨氮的吸附量通过公式 (1)得出:

$$
Q_{t}=\left(C_{0}-C_{t}\right) \frac{V}{W}
$$

式中, $Q_{t}$ 为磷和氨氮的吸附量, $\mathrm{mg} / \mathrm{g} ; C_{0}$ 为液相中初始磷和氨氮浓度, $\mathrm{mg} / \mathrm{L} ; C_{t}$ 为空白矫正后 $t$ 时刻的液相磷 和氨氮浓度, $\mathrm{mg} / \mathrm{L} ; V$ 为液体体积, $\mathrm{L} ; W$ 为样品质量, $\mathrm{g}$.

磷与氨氮去除率的计算公式为:

$$
\eta \%=\frac{\left(C_{0}-C_{t}\right)}{C_{0}} \times 100 \%
$$

式中, $\eta \%$ 为磷和氨氮的去除率, $\%$; $C_{0}$ 为液相中初始磷和氨氮浓度, $\mathrm{mg} / \mathrm{L} ; C_{t}$ 为空白矫正后 $t$ 时刻的液相磷和 氨氮浓度, $\mathrm{mg} / \mathrm{L}$.

本研究材料在吸附磷和氨氮过程中动力学行为采用准一级、二级动力学方程模型来进行研究, 准一级 
动力学方程为:

$$
Q_{t}=Q_{\mathrm{e}}\left(1-\mathrm{e}^{-K_{1} \cdot t}\right)
$$

式中, $Q_{\mathrm{e}}$ 为磷和氨氮浓度平衡时的平衡吸附量, $\mathrm{mg} / \mathrm{g} ; Q_{t}$ 为 $t(\mathrm{~h})$ 时刻的磷和氨氮吸附量, $\mathrm{mg} / \mathrm{g} ; K_{1}$ 为一级动 力学常数, $\min ^{-1}$.

准二级动力学方程为:

$$
\frac{T}{Q_{t}}=\frac{1}{K_{2} \cdot Q_{\mathrm{e}}^{2}}+\frac{t}{Q_{\mathrm{e}}}
$$

式中, $Q_{\mathrm{e}}$ 为磷和氨氮浓度平衡时的平衡吸附量, $\mathrm{mg} / \mathrm{g} ; Q_{t}$ 为 $t(\mathrm{~h})$ 时刻的磷和氨氮吸附量, $\mathrm{mg} / \mathrm{g} ; K_{2}$ 为二级动 力学常数, $\mathrm{g} /(\mathrm{mg} \cdot \mathrm{min})$.

吸附等温线通常采用经典的 Langmuir 和 Freundlich 吸附模型来进行绘制, 两者公式分别如 (5) 和 (6) 所示:

$$
\begin{gathered}
Q_{\mathrm{e}}=\frac{Q_{\mathrm{m}} \cdot K \cdot C_{\mathrm{e}}}{1+K \cdot Q_{\mathrm{m}}} \\
Q_{\mathrm{e}}=K_{f} \cdot C_{\mathrm{e}}^{\mathrm{e}}
\end{gathered}
$$

式中, $Q_{\mathrm{e}}$ 为磷和氨氮在液相中的平衡吸附量, $\mathrm{mg} / \mathrm{g} ; C_{\mathrm{e}}$ 为平衡吸附浓度, $\mathrm{mg} / \mathrm{L} ; Q_{\mathrm{m}}$ 为 Langmuir 理论最大吸附 量, $\mathrm{mg} / \mathrm{g} ; K$ 表示吸附剂对吸附质的亲和力, $\mathrm{L} / \mathrm{mg} ; K_{f}$ 为吸附系数, $\mathrm{L} / \mathrm{g} ; n$ 为常数, 通常用来表示吸附剂吸附强 度和表面的多相性.

热力学参数计算公式分别如 (7)、(8)、(9) 所示:

$$
\begin{gathered}
K_{D}=\frac{C_{0}-C_{\mathrm{e}}}{C_{\mathrm{e}}} \cdot \frac{V}{m} \\
\Delta G^{0}=-R \cdot T \ln \left(K_{D}\right) \\
\ln \left(K_{D}\right)=-\frac{\Delta H^{0}}{R \cdot T}+\frac{\Delta S^{0}}{R}
\end{gathered}
$$

式中, $\Delta G^{0}$ 为标准吉布斯自由能变, $\mathrm{kJ} / \mathrm{mol} ; \Delta S^{0}$ 为标准反应熵变, $\mathrm{kJ} /(\mathrm{mol} \cdot \mathrm{K}) ; \Delta H^{0}$ 为标准反应焓变, $\mathrm{kJ} /$ $\mathrm{mol} ; K_{D}$ 为固液分配系数, $\mathrm{ml} / \mathrm{g} ; R$ 为理想气体常数, $8.314 \mathrm{~J} /(\mathrm{mol} \cdot \mathrm{K}) ; T$ 为绝对温度, $\mathrm{K} ; m$ 为吸附剂质量, $\mathrm{g}$.

\section{2 结果与讨论}

\section{1 改性沉积物对磷和氨氮去除效果的评估}

如图 1 所示, 在相同温度下, 经氧化一载钠联合改性过的沉积物样品 (MS) 对磷和氨氮的去除效果均高 于原始沉积物材料 (S), 其中对磷的去除率均达到 $90 \%$ 以上. 在 $15^{\circ} \mathrm{C}$ 下, $\mathrm{S}$ 和 MS 两种材料对磷和氨氮的去 除率分别为 $34.3 \% 、 94.7 \%$ 和 $14 \% 、 86 \%$; 在 $25^{\circ} \mathrm{C}$ 下, 分别为 $27.0 \% 、 92.4 \%$ 和 $16 \% 、 89 \%$. 随着温度的升高, 两 种材料对氨氮的去除率均有提高, 相同材料对磷酸盐的去除率在 $25^{\circ} \mathrm{C}$ 时有小幅度下降; 当温度上升至 $35^{\circ} \mathrm{C}$ 时,改性材料对磷和氨氮的去除率有明显上升, 分别增加至 40\%、98\%和 20\%、90\%.

实验结果显示, 经改性后的沉积物材料较之原始材料, 对磷和氨氮的去除率均有大幅度提升. 首先, 经 $\mathrm{HNO}_{3}$ 氧化后, 沉积物材料比表面积可能增加, 表面活性点位数增多, 对磷和氨氮的吸附能力大大提高. 其 次, 经过负载 $\mathrm{Na}^{+}$, 一方面是在原沉积物表面负载一层活性较高物质, 增加材料比表面积, 另一方面也增加了 材料表面 $\mathrm{Na}_{2} \mathrm{O}$ 的含量 (表 2), 大幅度提高了对磷和氨氮的吸附能力. 实验结果同样表明, 温度对材料的吸 附性能有着重要影响. 当环境温度从 $15^{\circ} \mathrm{C}$ 增加到 $35^{\circ} \mathrm{C}$ 的过程中, 改性材料对磷和氨氮的去除率总体呈现上 升趋势, 这主要是由于在一定温度范围内升高温度, 会使分子运动加剧, 加快扩散速率, 增加了 $\mathrm{H}_{2} \mathrm{PO}_{4}^{-}$和 $\mathrm{NH}_{4}^{+}$和改性材料微粒表面的碰撞机会而导致吸附效果有所提高 ${ }^{[19]}$; 另一方面, 温度的升高使得更多的 $\mathrm{H}_{2} \mathrm{PO}_{4}^{-}$和 $\mathrm{NH}_{4}^{+}$获得能量进入到材料微粒表面而被吸附 ${ }^{[20]}$. 此外, 在 $25^{\circ} \mathrm{C}$ 时, 材料对 $\mathrm{H}_{2} \mathrm{PO}_{4}^{-}$的去除率略有下 降, 这可能是由于在材料的改性过程中, 采用 $\mathrm{HNO}_{3}$ 进行氧化改性, 引人了大量的阴离子, 在温度上升过程 中, 相应的阴离子与 $\mathrm{H}_{2} \mathrm{PO}_{4}^{-}$竞争活性吸附点位, 使得去除率略有下降. 因此, 在适当温度范围内, 提高温度有 利于改性材料对磷和氨氮的吸附. 


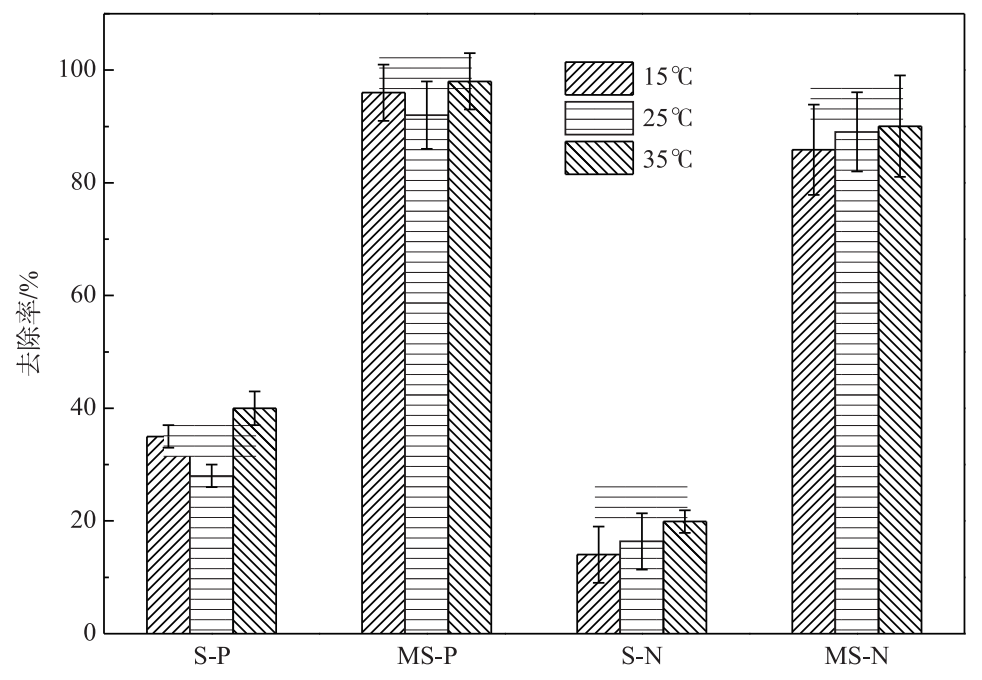

图 1 温度对改性材料氨氮和磷酸盐去除率的影响

(S-P 和 MS-P 分别为 $S$ 和 MS 对磷酸盐的去除率, S-N 和 MS-N 分别为 $S$ 和 MS 对氨氮的去除率, 下同)

Fig.1 Effect of temperature on ammonia nitrogen and phosphorus removal efficiency of modified materials

( S-P and MS-P for the efficiency of phosphorus using S and MS, respectively; S-N and MS-N for the efficiency of ammonia nitrogen using $\mathrm{S}$ and MS, respectively, the same below)

\section{2 改性沉积物对氮、磷吸附的动力学研究}

利用准一级和二级动力学方程 (公式 (3) 和 (4)) 拟合的两种材料在不同温度下对氮、磷吸附的动力学 曲线如图 2 所示.
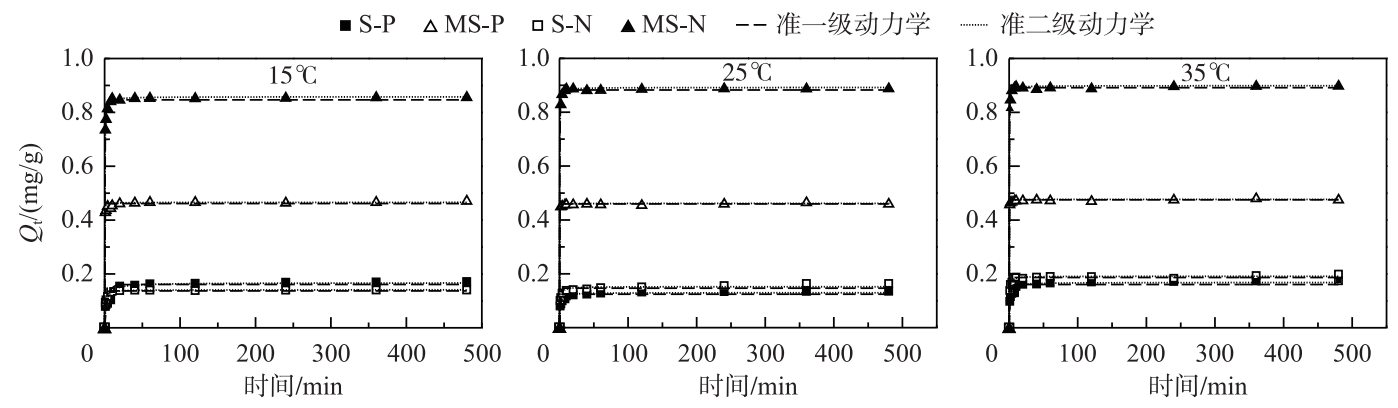

图 2 不同温度下两种材料对磷和氨氮的吸附动力学拟合曲线

Fig.2 Adsorption kinetics curves of phosphorus and ammonia nitrogen for two materials at different temperatures

结果表明, 在相同温度下, MS 对氨氮和磷的吸附量要高于 $\mathrm{S}$, 且两种材料在 $35^{\circ} \mathrm{C}$ 时对氨氮和磷的吸附 量均大于其他温度下的吸附量, $\mathrm{MS}$ 在 $35^{\circ} \mathrm{C}$, 初始 $\mathrm{P}$ 浓度为 $10 \mathrm{mg} / \mathrm{L}$ 的条件下, 对 $\mathrm{P}$ 的吸附量最大可达 0.48 $\mathrm{mg} / \mathrm{g}$, 在氨氮初始浓度为 $20 \mathrm{mg} / \mathrm{L}$ 的条件下, 对氨氮的吸附量最高达到 $0.891 \mathrm{mg} / \mathrm{g}$. 此外, 两种材料对氮、磷 的吸附量在 0 10 min 内迅速增加, $10 \mathrm{~min}$ 以后则呈现一个较为缓慢的吸附趋势. 约在 $40 \mathrm{~min}$ 时, 氨氮和磷 的吸附量达到最大值. 这主要是在吸附开始阶段由于静电作用导致 $\mathrm{H}_{2} \mathrm{PO}_{4}^{-}$和 $\mathrm{NH}_{4}^{+}$粘附在了材料表面, 使得 材料对氨氮和磷的吸附量快速上升. 接下来的吸附速率的下降是因为吸附点位都被占据,配位体的交换在 产生作用 ${ }^{[21]}$. 通过准一级、准二级动力学方程对两种材料的动力学曲线进行拟合, 所得参数如表 3 所示. 相 关系数 $R^{2}$ 的值表明准二级动力学方程能较好地描述两种材料对磷和氨氮的吸附动力学行为 $\left(R^{2}>0.93\right)$. 通 过准二级动力学方程拟合数据可知, 在 $15 、 25$ 和 $35^{\circ} \mathrm{C}$ 条件下, 改性材料 MS 对氨氮的平衡吸附量较高, 最大 
值可达 $0.891 \mathrm{mg} / \mathrm{g}$; 就 $\mathrm{P}$ 的平衡吸附量而言, 虽然其平衡吸附量 (最大值为 $0.474 \mathrm{mg} / \mathrm{g}$ ) 没有氨氮高, 但就其 他天然或改性后的材料而言 (石英砂和铝改性矾土磷吸附量分别为 0.02 和 $0.41 \mathrm{mg} / \mathrm{g}$ ) ${ }^{[22-23]}$, 改性后的沉积 物对 $\mathrm{P}$ 也有着较大的吸附性能. 此外, 表 3 中拟合参数显示了吸附速率常数 $K_{2}$ 的变化, 两种材料吸附氮、磷 的速率常数 $K_{2}$ 的值为 $\mathrm{MS}>\mathrm{S}$, 且环境温度越高, $K_{2}$ 值越大, 这主要是由于温度的升高, $\mathrm{Na}$ 与颗粒表面活性部 位络合能力更强, 更易与含氧官能团发生反应, 从而导致高温条件下, 氧化一载钠改性沉积物的吸附常数 较高.

表 3 运用准一级、准二级动力学方程拟合两种材料在不同温度下对磷和氨氮的吸附动力学参数

Tab.3 Fitting adsorption kinetics parameters of phosphorus and ammonia nitrogen at different temperatures for the two materials using pseudo-first-order and pseudo-second-order kinetic equations

\begin{tabular}{|c|c|c|c|c|c|c|c|}
\hline \multirow{2}{*}{ 温度 } & \multirow{2}{*}{ 材料样品 } & \multicolumn{3}{|c|}{ 准一级动力学 } & \multicolumn{3}{|c|}{ 准二级动力学 } \\
\hline & & $Q_{\mathrm{e}} /(\mathrm{mg} / \mathrm{g})$ & $K_{1} / \min ^{-1}$ & $R^{2}$ & $Q_{\mathrm{e}} /(\mathrm{mg} / \mathrm{g})$ & $K_{2} /(\mathrm{g} /(\mathrm{mg} \cdot \min ))$ & $R^{2}$ \\
\hline \multirow[t]{4}{*}{$15^{\circ} \mathrm{C}$} & S-P & $0.161 \pm 0.007$ & $0.246 \pm 0.055$ & 0.8550 & $0.162 \pm 0.005$ & $2.663 \pm 0.588$ & 0.9360 \\
\hline & MS-P & $0.462 \pm 0.003$ & $2.637 \pm 0.316$ & 0.9940 & $0.466 \pm 0.002$ & $22.595 \pm 3.663$ & 0.9980 \\
\hline & $\mathrm{S}-\mathrm{N}$ & $0.140 \pm 0.003$ & $0.891 \pm 0.110$ & 0.9627 & $0.141 \pm 0.001$ & $12.044 \pm 1.212$ & 0.9911 \\
\hline & MS-N & $0.846 \pm 0.006$ & $1.924 \pm 0.164$ & 0.9928 & $0.860 \pm 0.002$ & $6.722 \pm 0.351$ & 0.9994 \\
\hline \multirow[t]{4}{*}{$25^{\circ} \mathrm{C}$} & S-P & $0.124 \pm 0.004$ & $0.719 \pm 0.134$ & 0.9100 & $0.130 \pm 0.002$ & $8.783 \pm 1.327$ & 0.9750 \\
\hline & MS-P & $0.459 \pm 0.001$ & $3.358 \pm 0.254$ & 0.9990 & $0.461 \pm 0.001$ & $53.010 \pm 7.471$ & 0.9990 \\
\hline & $\mathrm{S}-\mathrm{N}$ & $0.147 \pm 0.004$ & $0.910 \pm 0.169$ & 0.9197 & $0.152 \pm 0.003$ & $9.925 \pm 1.790$ & 0.9696 \\
\hline & MS-N & $0.883 \pm 0.004$ & $2.140 \pm 0.138$ & 0.9968 & $0.892 \pm 0.002$ & $8.545 \pm 0.453$ & 0.9996 \\
\hline \multirow[t]{4}{*}{$35^{\circ} \mathrm{C}$} & S-P & $0.160 \pm 0.005$ & $0.685 \pm 0.132$ & 0.9050 & $0.168 \pm 0.003$ & $6.264 \pm 1.004$ & 0.9710 \\
\hline & MS-P & $0.474 \pm 0.001$ & $3.392 \pm 0.254$ & 0.9990 & $0.477 \pm 0.001$ & $53.080 \pm 7.474$ & 0.9990 \\
\hline & S-N & $0.187 \pm 0.002$ & $1.209 \pm 0.128$ & 0.9781 & $0.191 \pm 0.001$ & $13.338 \pm 1.346$ & 0.9938 \\
\hline & MS-N & $0.891 \pm 0.004$ & $2.362 \pm 0.149$ & 0.9976 & $0.899 \pm 0.002$ & $10.751 \pm 0.809$ & 0.9995 \\
\hline
\end{tabular}

\section{3 改性沉积物氮、磷吸附等温线分析}

经典 Langmuir 和 Freundlich 等温方程(式(5)和 (6)) 可用来探究材料对氨氮和磷的吸附机理. 本研究 通过对不同温度下的平衡数据进行非线性拟合, 进一步探究改性沉积物对氨氮和磷的吸附机制, 拟合结果 如图 3 所示, 拟合参数见表 4. 结果表明, 同一温度条件下, 随着溶液中磷和氨氮的平衡浓度的增加, 两种材 料对磷和氨氮的吸附量也分别随之增加. 根据表 4 中拟合数据 $R^{2}$ 显示, Langmuir 模型可以更好地描述吸附 等温线 (MS 对氮、磷拟合数据 $R^{2}>0.98$ ). 结果显示, 随着温度的升高, 两种材料对氨氮和磷的最大吸附量 $\left(Q_{\mathrm{m}}\right)$ 和亲和力常数 $K$ 值不断增大, 在 $35^{\circ} \mathrm{C}$ 时达到最高值, 分别为 $4.986 \mathrm{~L} / \mathrm{mg} 、 1.113 \mathrm{mg} / \mathrm{g}$ 和 $1.565 \mathrm{mg} / \mathrm{g}$ 、 $0.54 \mathrm{~L} / \mathrm{mg}$. 此外, 实验结果表明, 两种材料对氨氮和磷平衡吸附量随着溶液中氮、磷的平衡浓度的升高而增 加, 这主要与材料表面活性吸附点位数量有关, 平衡浓度越高, 表面活性吸附点位被占领速度越快, 平衡吸 附量也随之减小 ${ }^{[24]}$. 虽然 Langmuir 模型能较好地描述两种材料的吸附等温行为, 但 Freundlich 模型的 $n$ 值 亦可以反映材料吸附能力的强弱, $n$ 越小, 表明吸附质越容易被材料所吸附 ${ }^{[25]}$. 同时, Freundlich 模型中的 $K_{f}$ 值显示, MS 具有较高的 $K_{f}$ 值, 表明 MS 具有更大的吸附分配系数, 与磷和氨氮的结合能力较高, 从而促进材 料对磷和氨氮的吸附 ${ }^{[26]}$.

此外, 将本实验中改性材料的最大吸附量与其他氮、磷吸附剂进行了比较. 结果表明, 本研究中的 MS 对 磷的吸附量均达到常见的除磷吸附材料的吸附量 (如沸石 $0.26 \mathrm{mg} / \mathrm{g}$ 、陶粒砂 $0.51 \mathrm{mg} / \mathrm{g}$ 、石英砂 $0.02 \mathrm{mg} / \mathrm{g}$ 和碎岩石 $0.49 \mathrm{mg} / \mathrm{g}^{[27-28]}$. 与改性材料相比, 如磁黄铁矿和矾土 (吸附量分别为 0.62 和 $0.58 \mathrm{mg} / \mathrm{g}$ ), 也均有一 定竞争优势 ${ }^{[29]}$. 此外, 沸石对氨氮的去除研究已相对成熟, 部分研究成果也已市场化, 在工程应用领域, 沸 石对氨氮的吸附量可达 $4 \mathrm{~kg} / \mathrm{t}^{[30]}$. 本研究中改性黑臭河道沉积物对氨氮的吸附量虽低于沸石等材料, 但综 合考虑材料对磷酸盐和氨氮均有去除能力, 该材料可直接或间接应用于人工湿地填料、原位生态修复等领 域, 在一定程度上解决了黑臭河道沉积物资源化利用的问题, 因此, 该材料在磷和氨氮的去除上也具有一定 的应用前景. 

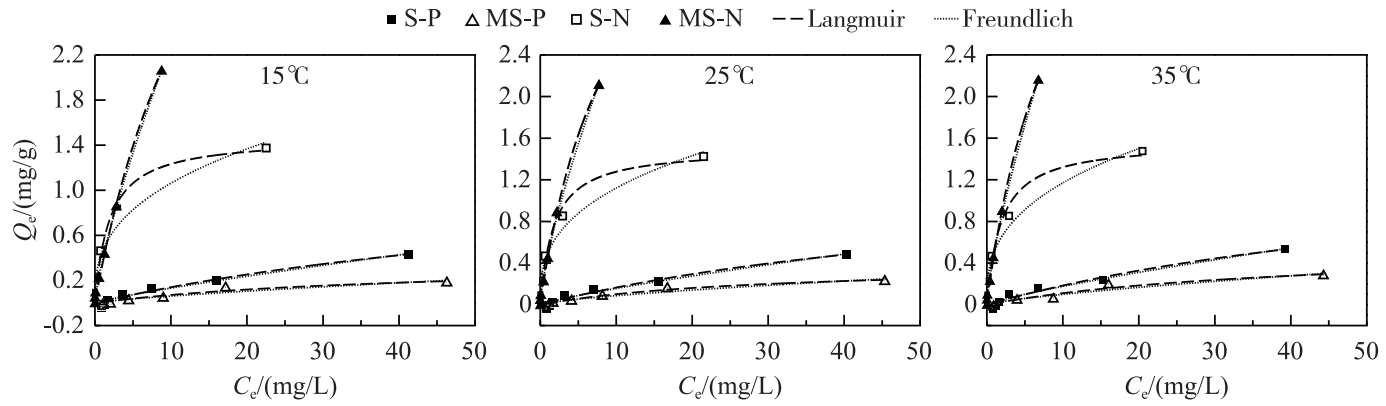

图 3 两种材料在不同温度下对磷和氨氮的吸附等温曲线

Fig.3 Adsorption isotherms of phosphorus and ammonia nitrogen for two materials at different temperatures

表 4 两种材料对磷的 Langmuir 和 Freundlich 吸附等温线常数

Tab.4 Adsorption isotherms parameters of phosphorus by Langmuir and Freundlich

\begin{tabular}{|c|c|c|c|c|c|c|c|}
\hline \multirow{2}{*}{ 温度 } & \multirow{2}{*}{ 材料样品 } & \multicolumn{3}{|c|}{ Langmuir } & \multicolumn{3}{|c|}{ Freundlich } \\
\hline & & $K /(\mathrm{L} / \mathrm{mg})$ & $Q_{\mathrm{m}} /(\mathrm{mg} / \mathrm{g})$ & $R^{2}$ & $K_{f} /(\mathrm{L} / \mathrm{g})$ & $n$ & $R^{2}$ \\
\hline \multirow[t]{4}{*}{$15^{\circ} \mathrm{C}$} & S-P & $0.012 \pm 0.009$ & $1.295 \pm 0.664$ & 0.9646 & $0.0189 \pm 0.007$ & $0.845 \pm 0.113$ & 0.9590 \\
\hline & MS-P & $0.522 \pm 0.076$ & $1.469 \pm 0.064$ & 0.9900 & $0.461 \pm 0.055$ & $0.363 \pm 0.043$ & 0.9570 \\
\hline & $\mathrm{S}-\mathrm{N}$ & $0.024 \pm 0.022$ & $0.366 \pm 0.188$ & 0.8764 & $0.011 \pm 0.008$ & $0.768 \pm 0.215$ & 0.8375 \\
\hline & MS-N & $0.065 \pm 0.012$ & $5.651 \pm 0.698$ & 0.9974 & $0.379 \pm 0.013$ & $0.781 \pm 0.017$ & 0.9990 \\
\hline \multirow[t]{4}{*}{$25^{\circ} \mathrm{C}$} & S-P & $0.014 \pm 0.01$ & $1.316 \pm 0.612$ & 0.9620 & $0.023 \pm 0.009$ & $0.816 \pm 0.109$ & 0.9580 \\
\hline & MS-P & $0.568 \pm 0.114$ & $1.503 \pm 0.087$ & 0.9830 & $0.497 \pm 0.047$ & $0.353 \pm 0.035$ & 0.9720 \\
\hline & $\mathrm{S}-\mathrm{N}$ & $0.035 \pm 0.019$ & $0.389 \pm 0.109$ & 0.9314 & $0.018 \pm 0.009$ & $0.692 \pm 0.152$ & 0.8838 \\
\hline & MS-N & $0.110 \pm 0.012$ & $4.603 \pm 0.308$ & 0.9984 & $0.473 \pm 0.021$ & $0.734 \pm 0.024$ & 0.9976 \\
\hline \multirow[t]{4}{*}{$35^{\circ} \mathrm{C}$} & S-P & $0.014 \pm 0.010$ & $1.498 \pm 0.765$ & 0.9580 & $0.027 \pm 0.010$ & $0.813 \pm 0.109$ & 0.9570 \\
\hline & MS-P & $0.540 \pm 0.126$ & $1.565 \pm 0.105$ & 0.9780 & $0.513 \pm 0.041$ & $0.357 \pm 0.030$ & 0.9810 \\
\hline & S-N & $0.025 \pm 0.016$ & $0.561 \pm 0.210$ & 0.9272 & $0.018 \pm 0.010$ & $0.736 \pm 0.151$ & 0.9005 \\
\hline & MS-N & $0.113 \pm 0.013$ & $4.986 \pm 0.368$ & 0.9984 & $0.517 \pm 0.018$ & $0.748 \pm 0.020$ & 0.9985 \\
\hline
\end{tabular}

\section{4 改性沉积物氮、磷吸附的热力学研究}

如图 4 所示, 根据公式 (9) 对 $\ln K_{\mathrm{D}}$ 和 $1000 / T$ 进行线性拟合, 可得 $\Delta H^{0} / R$ 和 $\Delta S^{0} / R$, 从而得出 $\Delta H^{0}$ 和 $\Delta S^{0}$. 各热力学参数见表 5. 通过公式(8) 得出标准吉布斯自由能变 $\Delta G^{0}$ 的值. $\Delta H^{0}$ 为正值, 表明吸附过程是个吸热 的过程, 更高的温度更有利于吸附 ${ }^{[31]}$. 此外, $\Delta S^{0}$ 为正值, 说明磷和氨氮倾向于吸附在改性材料颗粒表面 ${ }^{[32]}$. 一般来说, 随着固体表面微粒的吸附, 熵下降. 表 5 中显示, $\Delta G^{0}$ 为负值, 说明吸附行为是自发进行的 ${ }^{[33]}$. 然 而, 随着温度的升高, $\Delta G^{0}$ 下降, 说明在高温下, 更有利于改性材料对氮、磷的吸附 ${ }^{[34]}$. MS 的 $\Delta G^{0}$ 值更小, 说 明相比原始材料, MS 具有更高的吸附性能. 在同一温度下, 随着氮、磷初始浓度的增加, $\Delta G^{0}$ 值增大, 这表明 在氮、磷的吸附解吸过程中, 解吸比吸附过程更容易发生 ${ }^{[35]}$.

\section{3 结论}

1) 经过改性的材料对氨氮的去除率达到 $90 \%$ 以上,对磷的去除率达 $98 \%$ 以上.

2) 改性材料对磷的吸附量可达 $0.474 \mathrm{mg} / \mathrm{g}$, 其吸附量已高于一般的磷吸附材料, 在磷的去除上应用前 景较大.

3) 改性材料对氨氮的吸附量可达 $0.891 \mathrm{mg} / \mathrm{g}$, 其吸附量在一定程度上虽低于市面上氮吸附剂, 但综合 材料对磷的吸附量及黑臭河道沉积物资源化效率、经济成本等因素, 该材料在氨氮和磷的去除上也具有一 定的应用前景. 

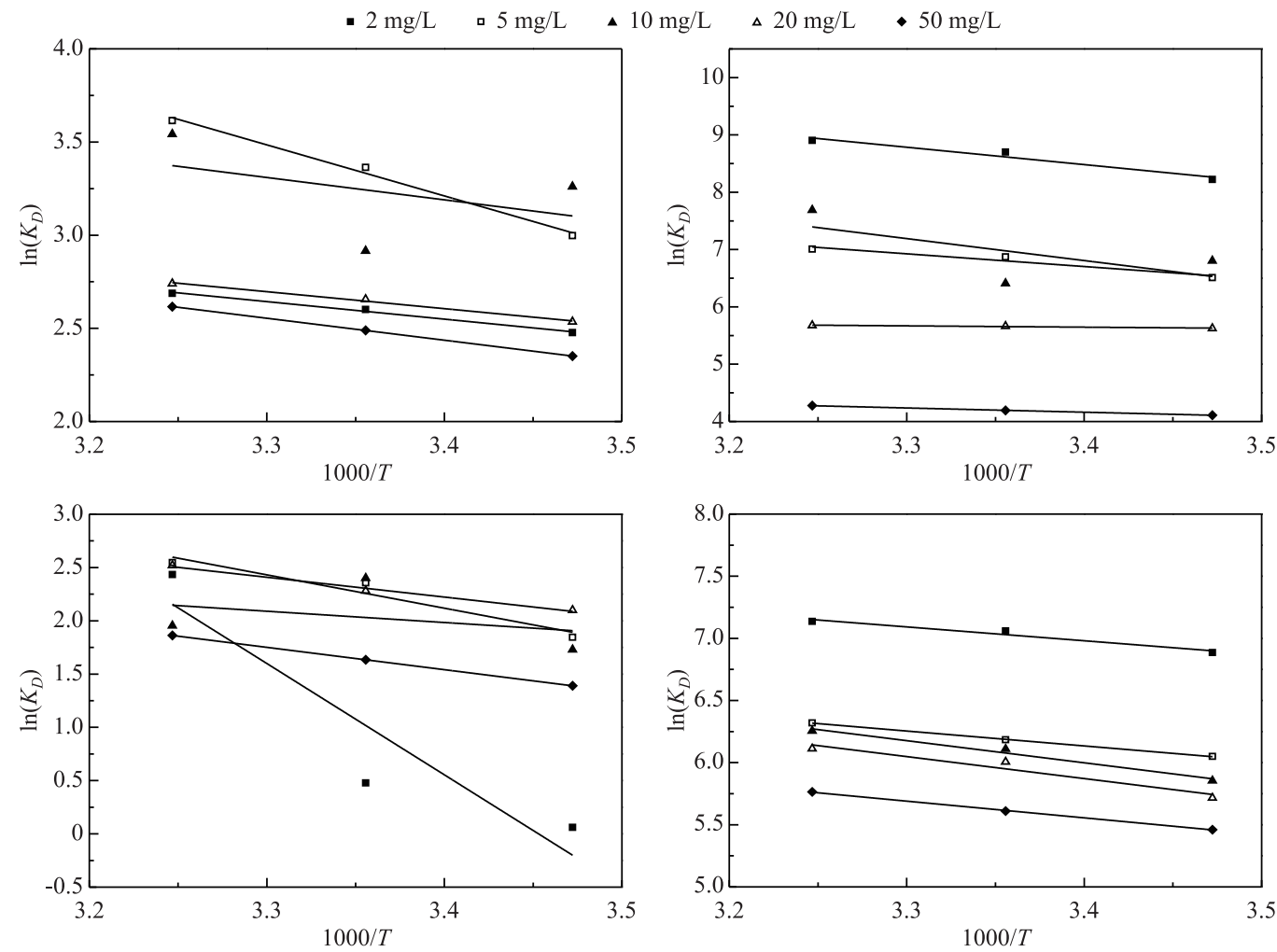

图 4 两种材料在不同初始浓度下吸附磷的热力学曲线

Fig.4 Thermodynamic curves of phosphorus adsorption by two materials at different initial concentrations

表 5 两种材料在不同初始浓度下吸附磷的热力学参数

Tab.5 Thermodynamic parameters of phosphorus adsorption by two materials at different initial concentrations

\begin{tabular}{|c|c|c|c|c|c|c|c|c|c|c|}
\hline \multirow{3}{*}{$\begin{array}{c}C_{0} / \\
(\mathrm{mg} / \mathrm{L})\end{array}$} & \multicolumn{5}{|c|}{ S-P } & \multicolumn{5}{|c|}{ MS-P } \\
\hline & \multirow{2}{*}{$\begin{array}{c}\Delta H^{0} / \\
(\mathrm{kJ} / \mathrm{mol})\end{array}$} & \multirow{2}{*}{$\begin{array}{c}\Delta S^{0} / \\
(\mathrm{kJ} /(\mathrm{mol} \cdot \mathrm{K}))\end{array}$} & \multicolumn{3}{|c|}{$\Delta G^{0} /(\mathrm{kJ} / \mathrm{mol})$} & \multirow{2}{*}{$\begin{array}{c}\Delta H^{0} / \\
(\mathrm{kJ} / \mathrm{mol})\end{array}$} & \multirow{2}{*}{$\begin{array}{c}\Delta S^{0} / \\
\mathrm{J} /(\mathrm{mol} \cdot \mathrm{K}))\end{array}$} & \multicolumn{3}{|c|}{$\Delta G^{0} /(\mathrm{kJ} / \mathrm{mol})$} \\
\hline & & & $15^{\circ} \mathrm{C}$ & $25^{\circ} \mathrm{C}$ & $35^{\circ} \mathrm{C}$ & & & $15^{\circ} \mathrm{C}$ & $25^{\circ} \mathrm{C}$ & $35^{\circ} \mathrm{C}$ \\
\hline 2 & 7.82 & 0.05 & -5.93 & -6.44 & -6.88 & 25.19 & 0.16 & -19.69 & -21.56 & -22.80 \\
\hline 5 & 22.78 & 0.10 & -7.18 & -8.33 & -9.26 & 18.46 & 0.12 & -15.59 & -17.03 & -17.95 \\
\hline 10 & 9.98 & 0.06 & -7.81 & -7.22 & -9.07 & 31.84 & 0.16 & -16.29 & -15.88 & -19.69 \\
\hline 20 & 7.57 & 0.05 & -6.07 & -6.58 & -7.02 & 1.91 & 0.05 & -13.47 & -14.03 & -14.54 \\
\hline 50 & 9.81 & 0.05 & -5.63 & -6.17 & -6.70 & 6.15 & 0.06 & -9.84 & -10.39 & -10.95 \\
\hline \multirow{3}{*}{$\begin{array}{c}C_{0} / \\
(\mathrm{mg} / \mathrm{L})\end{array}$} & \multicolumn{5}{|c|}{ S-N } & \multicolumn{5}{|c|}{ MS-N } \\
\hline & \multirow{2}{*}{$\begin{array}{c}\Delta H^{0} / \\
(\mathrm{kJ} / \mathrm{mol})\end{array}$} & \multirow{2}{*}{$\begin{array}{l}\Delta S^{0} / \\
(\mathrm{mol} \cdot \mathrm{K}))\end{array}$} & \multicolumn{3}{|c|}{$\Delta G^{0} /(\mathrm{kJ} / \mathrm{mol})$} & \multirow{2}{*}{$\begin{array}{c}\Delta H^{0} / \\
(\mathrm{kJ} / \mathrm{mol})\end{array}$} & \multirow{2}{*}{$\begin{array}{c}\Delta S^{0} / \\
\mathrm{J} /(\mathrm{mol} \cdot \mathrm{K}))\end{array}$} & \multicolumn{3}{|c|}{$\Delta G^{0} /(\mathrm{kJ} / \mathrm{mol})$} \\
\hline & & & $15^{\circ} \mathrm{C}$ & $25^{\circ} \mathrm{C}$ & $35^{\circ} \mathrm{C}$ & & & $15^{\circ} \mathrm{C}$ & $25^{\circ} \mathrm{C}$ & $35^{\circ} \mathrm{C}$ \\
\hline 2 & 86.88 & 0.30 & -0.14 & -1.18 & -6.23 & 9.27 & 0.09 & -16.49 & -17.49 & -18.27 \\
\hline 5 & 26.01 & 0.11 & -4.42 & -5.84 & -6.52 & 9.96 & 0.08 & -14.49 & -15.32 & -16.18 \\
\hline 10 & 8.80 & 0.05 & -4.14 & -5.95 & -5.01 & 14.81 & 0.10 & -14.02 & -15.14 & -16.02 \\
\hline 20 & 15.47 & 0.07 & -5.03 & -5.65 & -6.45 & 14.71 & 0.10 & -13.69 & -14.88 & -15.65 \\
\hline 50 & 17.43 & 0.07 & -3.33 & -4.05 & -4.77 & 11.17 & 0.08 & -13.08 & -13.90 & -14.76 \\
\hline
\end{tabular}


4) 通过对改性材料氮、磷去除机制的研究,发现该改性材料对氮、磷的去除效果受温度影响,较高的温 度更有利于材料对氮、磷的去除. 这也表明在气候温度较高、氮磷含量较高的水体更适合采用该改性材料去 除氮、磷.

5) 本研究中的改性沉积物可以被认为是在污染水体净化领域的高效去除营养盐材料,同时本研究结果 为黑臭河道沉积物资源化途径和水体中氮、磷削减提供了一定的理论依据.

\section{4 参考文献}

[ 1 ] He Y, Shen SY, Huang MS et al. Study on nitrification-denitrification in sediment of urban black and smelly river. Journal of Ecological Environment, 2012, 21(6) : 1166-1170. [何岩, 沈叔云, 黄民生等. 城市黑臭河道底泥内源氮硝化一 反硝化作用研究. 生态环境学报, 2012, 21(6): 1166-1170.]

[ 2 ] Cao CJ, Chen ZL, Wang J et al. Review of sediment ecological dredging in urban black-odors river treatment. Journal of East China Normal University: Natural Science Edition, 2011, 1(1): 32-42. [曹承进, 陈振楼, 王军等. 城市黑臭河 道底泥生态疏浚技术进展. 华东师范大学学报: 自然科学版, 2011, 1(1) : 32-42.]

[ 3 ] Dong WY, Luo Y, Liu TZ et al. Discussion on disposal technology of river contaminated sediment: application to comprehensive improvement project of main-stream of Longganghe River. Water Conservancy and Hydropower Technology, 2012, 43(8) : 5-8. [ 董文艺, 罗雅, 刘泮宙等. 河道污染底泥处理技术探讨一一在龙岗河干流综合治理工程中应用. 水 利水电技术, 2012, 43(8): 5-8.]

[ 4 ] Liu CJ, Li YZ, Luan ZK et al. Adsorption removal of phosphate from aqueous solution by active red mud. Journal of Environmental Sciences-China, 2007, 19(10): 1166-1170.

[ 5 ] Li D, Min H, Jiang X et al. One-pot synthesis of aluminum-containing ordered mesoporous silica MCM-41 using coal fly ash for phosphate adsorption. Journal of Colloid and Interface Science, 2013, 404: 42-48.

[ 6 ] Liu DH, Li YP, Li TG et al. Progress and development direction of marine sediment restoration technology. Environmental Science and Technology, 2017, 40(S1) : 150-156. [刘大海, 李彦平, 李铁刚等. 海洋沉积物修复技术进展及发展方 向初探. 环境科学与技术, $2017, \mathbf{4 0}(\mathrm{S} 1)$ : 150-156.]

[ 7 ] Schindle DW. Eutrophication and recovery in experimental lakes-implications for lake management. Science, $1974, \mathbf{1 8 4}$ (4139) : 897-899.

[ 8 ] Conley DJ, Paerl HW, Howarth RW et al. Ecology controlling eutrophication: nitrogen and phosphorus. Science, 2009, 323(5917) : 1014-1015.

[ 9 ] Schindler DW, Hecky RE. Eutrophication: more nitrogen data needed. Science, 2009, 324(5928) : 721-722.

[10] Lin J, Zhan Y, Zhu Z. Evaluation of sediment capping with active barrier systems (ABS) using calcite/zeolite mixtures to simultaneously manage phosphorus and ammonium release. Science of the Total Environment, 2011, 409(3) : 638-646.

[11] Sakadevan K, Bavor HJ. Phosphate adsorption characteristics of soils, slags and zeolite to be used as substrates in constructed wetland systems. Water Research, 1998, 32(4) : 393-399.

[12] George M, Ayoub B. Koopman et al. Iron and aluminum hydroxy (oxide) coated filter media for low-concentration phosphorus removal. Water Environment Research, 2001, 73(4) : 478-485.

[13] Bouzid J, Elouear Z, Ksibi M et al. A study on removal characteristics of copper from aqueous solution by sewage sludge and pomace ashes. Journal of Hazardous Materials, 2008, 152(2) : 838-845.

[ 14] Huang W, Zhang L, Gao J et al. Removal of dissolved inorganic phosphorus with modified gravel sand: kinetics, equilibrium, and thermodynamic studies. Desalination and Water Treatment, 2016, 57(7) : 3074-3084.

[15] Ruban V, Lopez-Sanchez JF, Pardo P et al. Development of a harmonised phosphorus extraction procedure and certification of a sediment reference material. Journal of Environmental Monitoring, 2001, 3(1) : 121-125.

[16] Wang SR, Jin XC, Jiao LX. Distribution oftransferable nitrogen in different grain size from the different trophic level lake sediments. Environmental Science Research, 2007, 20(3): 52-57. [王圣瑞, 金相灿, 焦立新. 不同污染程度湖泊沉积物 中不同颗粒级可转化态氮分布. 环境科学研究, 2007, 20(3) : 52-57.]

[17] Liao X, Zhang C, Yao L et al. Sorption behavior of nonylphenol ( NP) on sewage-irrigated soil: kinetic and thermodynamic studies. Science of the Total Environment, 2014, 473/474(4) : 530-536.

[18] Huang W, Lu Y, Li JH et al. Effect of ionic strength on phosphorus sorption in different sediments from a eutrophic plateau 
lake. RSC Advances, 2015, 5(97) : 79607-79615.

[19] Kostura B, Kulveitova H, Lesko J. Blast furnace slags as sorbents of phosphate from water solutions. Water Research, $2005,39(9):$ 1795-1802.

[20] Ye H, Chen F, Sheng Y et al. Adsorption of phosphate from aqueous solution onto modified palygorskites. Separation and Purification Technology, 2006, 50(3): 283-290.

[21] Ding C, Yang X, Liu W et al. Removal of natural organic matter using surfactant modified iron oxide-coated sand. Journal of Hazardous Materials, 2010, 174 (3) : 567-572.

[22] Drizo A, Frost CA, Grace J et al. Physico-chemical screening of phosphate-removing substrates for use in constructed wetland systems. Water Research, 1999, 33(17) : 3595-3602.

[23] Li RH, Kelly C, Keegan R et al. Phosphorus removal from wastewater using natural pyrrhotite. Colloids and Surfaces aPhysicochemical and Engineering Aspects, 2013, 427(1): 13-18.

[24] Liu J, Wan L, Zhang L et al. Effect of $\mathrm{pH}$, ionic strength, and temperature on the phosphate adsorption onto lanthanumdoped activated carbon fiber. Journal of Colloid and Interface Science, 2011, 364 (2) : 490-496.

[25] Fouladi Tajar A, Kaghazchi T, Soleimani M. Adsorption of cadmium from aqueous solutions on sulfurized activated carbon prepared from nut shells. Journal of Hazardous Materials, 2009, 165 (1/2/3) : 1159-1164.

[26] Kumar PS, Ramalingam S, Senthamarai C et al. Adsorption of dye from aqueous solution by cashew nut shell: Studies on equilibrium isotherm, kinetics and thermodynamics of interactions. Desalination, 2010, 261 (1) : 52-60.

[27] Drizo A, Frost CA, Grace J et al. Physico-chemical screening of phosphate-removing substrates for use in constructed wetland systems. Water Research, 1999, 33(17) : 3595-3602.

[28] Arias CA, Del Bubba M, Brix H. Phosphorus removal by sands for use as media in subsurface flow constructed reed beds. Water Research, 2001, 35(5): 1159-1168.

[29] Li RH, Kelly C, Keegan R et al. Phosphorus removal from wastewater using natural pyrrhotite. Colloids and Surfaces aPhysicochemical and Engineering Aspects, 2013, 427(1): 13-18.

[30] Rao L, Wang XJ. Pilot study on the treatment of ammonia nitrogen wastewater by natural zeolite. Water Treatment Technolo$g y, 2016$, (4) : 104-106. [ 饶力, 汪晓军. 天然沸石处理氨氮废水的中试研究. 水处理技术, 2016, (4) : 104-106.]

[31] Alagumuthu G, Rajan M. Equilibrium and kinetics of adsorption of fluoride onto zirconium impregnated cashew nut shell carbon. Chemical Engineering Journal, 2010, 158 (3) : 451-457.

[32] Huang W, Zhang L, Gao J et al. Removal of dissolved inorganic phosphorus with modified gravel sand: kinetics, equilibrium, and thermodynamic studies. Desalination and Water Treatment, 2016, 57 (7) : 3074-3084.

[33] Huang LD, Fu L, Jin CW et al. Effect of temperature on phosphorus sorption to sediments from shallow eutrophic lakes. Ecological Engineering, 2011, 37 (10): 1515-1522.

[34] Li KQ, Zheng Z, Huang XF et al. Equilibrium, kinetic and thermodynamic studies on the adsorption of 2-nitroaniline onto activated carbon prepared from cotton stalk fibre. Journal of Hazardous Materials, 2009, 166 (1) : 213-220.

[35] Kim D. Adsorption characteristics of Fe ( III ) and Fe ( III )-NTA complex on granular activated carbon. Journal of Hazardous Materials, 2004, 106 (1): 67-84. 